\title{
Additional Resection of the Pancreas Body Prevents Postoperative Pancreas Fistula in Patients with Portal Annular Pancreas Who Undergo Pancreaticoduodenectomy
}

\author{
Jun Muto Yohei Mano Noboru Harada \\ Hideaki Uchiyama Tomoharu Yoshizumi \\ Akinobu Taketomi Ken Shirabe Yoshihiko Maehara \\ Department of Surgery and Science, Graduate School of Medical Sciences, \\ Kyushu University, Fukuoka, Japan
}

\section{Key Words}

Portal annular pancreas - Pancreaticoduodenectomy - Pancreas fistula

\begin{abstract}
Portal annular pancreas (PAP) is a rare variant in which the uncinate process of the pancreas extends to the dorsal surface of the pancreas body and surrounds the portal vein or superior mesenteric vein. Upon pancreaticoduodenectomy (PD), when the pancreas is cut at the neck, two cut surfaces are created. Thus, the cut surface of the pancreas becomes larger than usual and the dorsal cut surface is behind the portal vein, therefore pancreatic fistula after PD has been reported frequently. We planned subtotal stomach-preserving PD in a 45-year-old woman with underlying insulinoma of the pancreas head. When the pancreas head was dissected, the uncinate process was extended and fused to the dorsal surface of the pancreas body. Additional resection of the pancreas body $1 \mathrm{~cm}$ distal to the pancreas tail to the left side of the original resection line was performed. The new cut surface became one and pancreaticojejunostomy was performed as usual. No postoperative complications such as pancreatic fistula occurred. Additional resection of the pancreas body may be a standardized procedure in patients with PAP in cases of pancreas cut surface reconstruction.
\end{abstract}




\section{Introduction}

Portal annular pancreas (PAP) is a rare variant in which the uncinate process of the pancreas extends to the dorsal surface of the pancreas body and surrounds the portal vein or superior mesenteric vein (SMV) [1]. PAP is also called circumportal pancreas [2]. Recent progress has made it possible to diagnose PAP preoperatively with contrast-enhanced multidetector computed tomography (MDCT) or magnetic resonance imaging [2]. Nevertheless, PAP is still not recognized as an important finding in cases of pancreas cut surface reconstruction. Most cases of PAP have been reported during pancreaticoduodenectomy (PD) [1-5]. Upon PD, when the pancreas is cut at the neck, which is located at the left side of the portal vein, two cut surfaces of the pancreas are created. Thus, the cut surface of the pancreas becomes larger than usual and the dorsal cut surface is behind the portal vein, therefore pancreatic fistula after PD has been reported frequently $[2,5]$.

Here, we report a patient with PAP who underwent subtotal stomach-preserving PD (SSPPD) and additional pancreas resection $1 \mathrm{~cm}$ distal to the pancreas tail to the left side of the original resection line. This allowed us to perform safe pancreatic jejunal anastomosis.

\section{Case Report}

A 45-year-old woman became unconscious with shivering during exercising. She was taken to hospital by ambulance. Her unconsciousness was due to hypoglycemia and she recovered quickly after intravenous administration of glucose. For about 2 years, she had been aware of palpitations, cold sweat and nausea that improved after consuming sweets during exercise. CT was performed and a $2-\mathrm{cm}$ nodule in the pancreas head was revealed. She was admitted to the Department of Surgery and Science, Kyushu University with suspicion of insulinoma of the pancreas head.

Complete blood cell count was normal; immunoreactive insulin level was $178 \mu \mathrm{U} / \mathrm{ml}$ and fasting blood sugar $56 \mathrm{mg} / \mathrm{dl}$. Serum levels of glucagon, gastrin, growth hormone, adrenocorticotropic hormone, thyroid-stimulating hormone and free T4 were within normal limits. The tumor markers pro-gastrin-releasing peptide, neuron-specific enolase, carcinoembryonic antigen and carbohydrate antigen 19.9 were also within normal limits. Glucagon tosylate examination was performed and Fajan's index was 3.84. On contrast-enhanced MDCT, an enhanced 2-cm nodule was detected in the pancreas head. We diagnosed her condition as insulinoma of the pancreas head.

We planned to treat her with SSPPD. When the pancreas head was dissected at the usual level, which was to the left of the portal vein, the uncinate process was extended and fused to the dorsal surface of the pancreas body. Pancreatic duct-like structures were observed in the usual place and the extended uncinate process. Reconstruction of the pancreas cut surface seemed to be difficult, and additional resection of the pancreas body $1 \mathrm{~cm}$ distal to the pancreas tail to the left side of the original resection line was performed. On the new cut surface, there was only one pancreatic duct in the usual place and pancreaticojejunostomy was performed as usual.

After the operation, MDCT revealed an extended uncinate process of the pancreas that was fused to the dorsal surface of the pancreas body by surrounding the portal vein (fig. 1). The maximum length of the fused lesion was $10 \mathrm{~mm}$ in transverse section. No postoperative complications such as pancreatic fistula occurred, and the patient was discharged from hospital on day 22 after the operation. 


\section{Discussion}

PAP is an anatomical variant in which the uncinate process extends and joins the dorsal area of the pancreas body. The portal vein and/or the SMV are surrounded by the uncinate process of the pancreas [1]. Karasaki et al. [3] reviewed 700 abdominal contrast-enhanced MDCT scans, irrespective of the patients' disease and sex, and diagnosed 8 cases $(1.14 \%)$ of PAP. There are no clinical symptoms related to PAP, and it emerges only when operative intervention is required.

Joseph et al. [4] proposed another PAP classification that categorizes cases according to the location of the main pancreatic duct. The main pancreatic duct is posterior to the portal vein because the ventral bud of the pancreas fuses with the dorsal area of the pancreas body in type I PAP. Type II PAP is associated with pancreas divisum and the main pancreatic duct is posterior to the portal vein. Type III PAP is when the uncinate process alone is involved in encasement of the portal vein and/or SMV. Our case was type II according to this classification. Sugiura et al. [1] reported the first case of PAP in 1987. To the best of our knowledge, 5 cases of PAP in patients who have undergone PD have been reported to date [1-5].

Reconstruction of the pancreas cut surface in PD is difficult and crucial in PAP for the following reasons: (1) the cut surface of the pancreas is larger than usual, (2) the dorsal cut surface of the pancreas is located in the dorsal portal vein, and (3) a pancreatic duct can be observed in the uncinate process. The standard operative procedure for reconstruction of the pancreatic cut surface in PAP has not been determined to date. Sugiura et al. [1] closed the pancreas cut surface by suture when it was dorsal to the SMV. Mizuma et al. [5] sutured the pancreas body by mattress suture to make the cutting surface narrower. Karasaki et al. [3] divided the fused region of the uncinate process and dorsal area of the pancreas body.

Pancreatic fistula occurred in 2 of 4 cases with PD that had information about postoperative complications. Closure of the dorsal cut surface by suture should be avoided in patients with type I and II PAP according to the classification of Joseph et al. [4], because the main pancreatic duct is located at the dorsal cut surface. Even when the main pancreatic duct is found at the dorsal pancreas cut surface in patients with type I and II PAP [4], anastomosis of the pancreatic duct and jejunum is technically difficult because fine anastomosis has to be done behind the portal vein.

Additional resection of the pancreas body to the left of the original resection line seems to be reasonable and can make the cutting surface the same as usual anatomically, although this method has not been reported previously. By adding further resection, only one pancreatic duct is observed on the cutting surface and the portal vein never interferes with reconstruction. According to Karasaki et al. [3], the mean length of the fusion between the lingual projection and the body of the pancreas was $9.4 \mathrm{~mm}$, therefore an additional $1-\mathrm{cm}$ resection of the pancreas body may be sufficient for safe anastomosis (fig. 2 ).

In conclusion, additional resection of the pancreas body may be a standardized procedure in patients with PAP who are undergoing reconstruction of the pancreas cut surface. 


\section{Disclosure Statement}

J. Muto and the other co-authors have no conflict of interest.

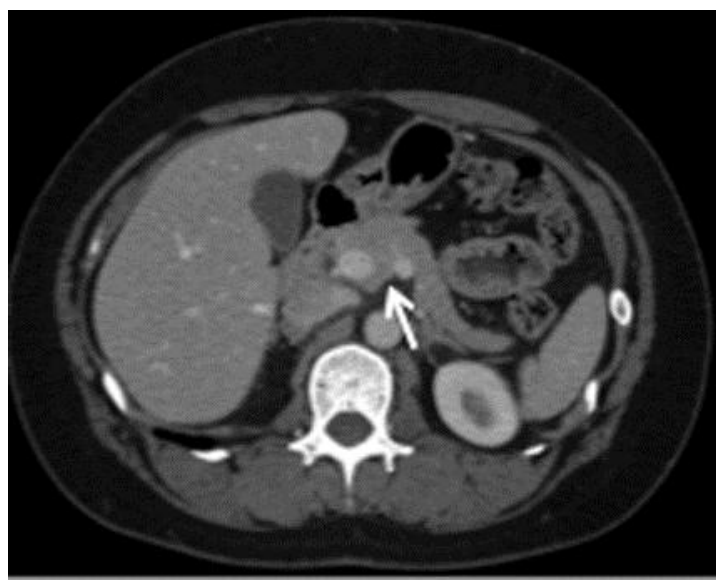

Fig. 1. Preoperative CT showed that the uncinate process of the pancreas extended and fused to the dorsal surface of the pancreas body by surrounding the portal vein (arrow).

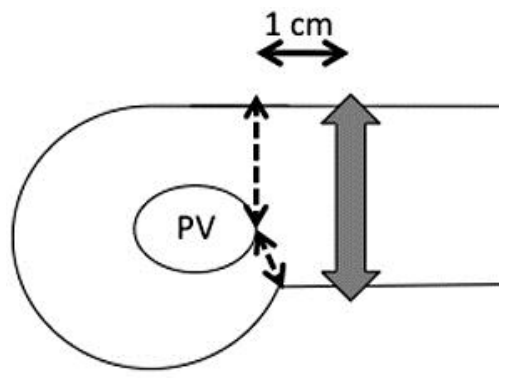

Fig. 2. Schematic representation of the pancreas. Additional resection of the pancreas body $1 \mathrm{~cm}$ to the left of the portal vein seems to be reasonable and can make the cutting surface the same as usual anatomically.

\section{References}

1 Sugiura Y, Shima S, Yonekawa H, Yoshizumi Y, Ohtsuka H, Ogata T: The hypertrophic uncinate process of the pancreas wrapping the superior mesenteric vein and artery. Jpn J Surg 1987;17:182-185.

2 Hamanaka Y, Evans J, Sagar G, Neoptolemos J: Complete pancreatic encasement of the proximal hepatic portal vein: a previously undescribed congenital anomaly. Br J Surg 1997;84:785.

-3 Karasaki H, Mizukami Y, Ishizaki A, et al: Portal annular pancreas, a notable pancreatic malformation: frequency, morphology, and implications for pancreatic surgery. Surgery 2009;146:515-518.

4 Joseph P, Raju RS, Vyas FL, Eapen A, Sitaram V: Portal annular pancreas. A rare variant and a new classification. J Pancreas (Online) 2010;11:453-455.

5 Mizuma M, Suzuki M, Umino R, Katayori T, Rikiyama T, Takeuchi H, Shiroai S, Matsuno M: A case of 'portal annular pancreas' on that the ventral pancreas joins with the pancreatic body encircling the portal vein. Tan to Sui 2001;22:963-966. 